\title{
Rendimiento Académico en Estudiantes de Ingeniería Comercial: Modelo por Competencias y Factores de Influencia
}

\author{
Catherine A. Avendaño, Karol A. Gutiérrez, Camila F. Salgado y Manuel Alonso-Dos-Santos* \\ Universidad Católica de la Santísima Concepción, Facultad de Ciencias Económicas y Administrativas. \\ Alonso de Rivera 2850, Concepción-Chile (e-mail: cavendano@icomercial.ucsc.cl; \\ kgutierrez@icomercial.ucsc.cl; csalgado@icomercial.ucsc.cl; malonso@ucsc.cl) \\ * Autor a quien debe ser dirigida la correspondencia
}

Recibido Dic. 2, 2015; Aceptado Feb. 8, 2016; Versión final Feb. 11, 2016, Publicado Jun. 2016

\begin{abstract}
Resumen
Se ha estudiado cómo ha influido en el rendimiento académico de los estudiantes la implementación del modelo de formación por competencias, tomando como base el modelo anterior por contenidos. Para esto se utilizó una base de datos de 852 alumnos matriculados durante los años 2007-2014 en la carrera de Ingeniería Comercial de la Universidad Católica de la Santísima Concepción en Chile. Se aplicó un modelo de regresión lineal, donde la variable dependiente fue el promedio acumulado de los alumnos al primer año Se concluye que aquellos alumnos que estudian bajo el modelo por competencia obtienen un mejor rendimiento académico en relación a los que estudian bajo la formación tradicional.
\end{abstract} académico

\section{Academic Performance in Business Studies: Competency- Based Education and Influencing Factors}

\begin{abstract}
A study has been done on the how academic performance of the students has been influenced by the implementation of competency-based training model based on the traditional model. For this investigation a database of 852 enrolled students during the years 2007-2014 in the career Ingeniería Comercial of the Universidad Católica de la Santísima Concepción in Chile, was used. This investigation has a purpose to answer a question, for that reason it was estimating a model with a regression line. The dependent variable was the cumulative average in their first year of the career. It is concluded that those students that study in the competency-based training model obtain better academic performance in relation to students that follow the traditional model.
\end{abstract}

Keywords: curricular model; training by content; competency-based training model; academic performance 


\section{INTRODUCCIÓN}

Producto de la globalización, del avance científico y tecnológico, la educación superior se ha visto enfrentada a una serie de cambios en las últimas décadas. La UNESCO (1998) propone que la educación superior sea reformada y alineada hacia un enfoque centrado en el estudiante, que responda a los requerimientos del trabajo y desarrollo de las naciones. Estos cambios en la educación superior abarcan diversos aspectos del proceso de formación, que son traducidos en el modelo curricular.

El modelo curricular es una propuesta que detalla la metodología y el diseño de todos los componentes incluidos en la configuración del profesional como contenidos impartidos, tipo de evaluación, objetivos a lograr en los estudiantes, entre otros (Vidal y Pernas, 2007). En este sentido, podemos encontrar en la literatura educativa autores que proponen diversos modelos para el diseño curricular, Vélez y Terán (2009) hacen referencia al modelo tradicional de Tyler (1960), modelo vínculo sociedad-individuo de Taba (1974), modelo de enfoque práctico de Schwab (1983), modelo práctico-profesional de Walker (1990) y el diseño curricular de Arnaz (1993). Por otro lado, Riesco (2008) se refiere al modelo de formación por competencias del Espacio Europeo de la Educación Superior (EEES) formulado en el año 1999. El modelo del EEES está fundamentado en el aprendizaje basado en competencias, enfocado en desarrollar competencias genéricas y específicas que nacen de requerimientos esenciales de cada profesión, combinando la teoría y práctica, donde el profesorado toma un rol redefinido (Palominos, et al., 2014), y el estudiante un rol activo en el proceso enseñanza-aprendizaje (Villa y Poblete, 2007).

Las universidades Chilenas aplicaron por varios años el modelo tradicional basado en contenidos (CRUCH, 2012), el que comenzó a presentar deficiencias ante las nuevas exigencias del entorno y sus participantes, generando dificultades como la transmisión de los conocimientos teóricos y el desarrollo de nuevas capacidades del profesional. Como una solución a estas problemáticas se incorporó el modelo basado en competencias, que entregaría mayor eficiencia y calidad en el proceso de aprendizaje y daría respuesta a las demandas de la sociedad (CRUCH, 2012). Tras más de una década de armonización y homologación de competencias transversales y profesionales en Chile y el resto del mundo (Bustamante et al., 2015), el nuevo modelo de formación basado en competencias modifica la estructura curricular pasando de un enfoque basado en el logro de objetivos a uno basado en la formación de competencias (Tardif, 2003), lo que genera un enfoque pedagógico centrado en el estudiante (Tejada, 2002). En contraste, los modelos catalogados como tradicionales en Chile se han caracterizado principalmente según el informe de la OCDE (2009) por currículos rígidos, programas de formación muy extensos, y métodos de enseñanza y aprendizaje evaluados como tradicionales y centrados en la trasmisión mecánica de contenidos (Guzmán, et al., 2015). El modelo basado en competencias, en contraposición al modelo tradicional, se centra en el aprendizaje. Sus diferencias entre otras se basan en la evaluación, el método docente y en el enfoque de las competencias. La tabla 1 resume las comparaciones al respecto (Fuente: Martínez-Clares, et al., 2008).

Tabla 1: Comparación de la formación tradicional con la formación basada en competencias

\begin{tabular}{|c|c|}
\hline Formación tradicional & Formación basada en competencias \\
\hline Desconectada de la realidad del entorno. & $\begin{array}{l}\text { Atenta a las necesidades cambiantes de la } \\
\text { sociedad y sus profesionales. }\end{array}$ \\
\hline Enfoque centrado en la enseñanza. & $\begin{array}{l}\text { Enfoque centrado en el aprendizaje y la gestión } \\
\text { del conocimiento. }\end{array}$ \\
\hline Prima la transferencia de información. & Importancia de la formación integral y permanente. \\
\hline $\begin{array}{l}\text { Desconocimiento de los intereses de los estudiantes y de la } \\
\text { necesidad de potenciar sus capacidades y habilidades. }\end{array}$ & $\begin{array}{l}\text { Se parte de la necesidad de potenciar las } \\
\text { competencias genéricas, transversales y } \\
\text { específicas de los colectivos a los que va dirigida. }\end{array}$ \\
\hline Currículo compartamentalizado y poco flexible & Currículo integrado y flexible \\
\hline Clase magistral como metodología única & Metodología diversa, activa y participativa. \\
\hline Alumno receptor pasivo de información. & Alumno agente de su propio aprendizaje. \\
\hline Uso de texto escrito como prioritario. & Centrada en otras formas alternativas de trabajo. \\
\hline
\end{tabular}

En virtud de lo anterior, creemos que es importante conocer cómo ha influido el cambio de modelo de enseñanza en los estudiantes. Junto con los factores género, edad, preferencia en elección de la enseñanza, educación de los progenitores y tipo de establecimiento educativo previo, esperamos examinar la influencia sobre el rendimiento académico. 
Navarro (2003) describe el rendimiento académico como la expresión de las habilidades, actitudes y valores que son desarrollados por el alumno a través del proceso de enseñanza-aprendizaje, es decir son todas aquellas acciones dirigidas a la explicación e interpretación de lo aprendido y que se sintetizan en valores cuantitativos o cualitativos. Con respecto a la evidencia empírica sobre el rendimiento académico se destaca la teoría de los determinantes del rendimiento académico de Tejedor (2003), quien realiza una investigación científica de más de tres años en una universidad de Salamanca, donde reúne diferentes estudios de diversos autores y concluye que son alrededor de 12 factores que explican el rendimiento académico, agrupándolos en cinco categorías: variables de identificación, psicológicas, académicas, pedagógicas y socio familiares. De forma similar, nos encontramos con diversos estudios que hacen referencia a los factores antes mencionados y que influyen en el rendimiento académico de los alumnos. Como antecedentes para introducir las hipótesis, examinamos algunas investigaciones previas.

En referencia al género, Soria y Zúñiga (2014) encontraron una relación directa con el rendimiento de los alumnos, siendo las mujeres quienes obtienen un mayor promedio. Otras investigaciones encontraron resultados dispares. Por ejemplo, Borde (1998) no encontró diferencias, y en cambio Woodfield y Earl-Novell (2006) coinciden con Soria y Zúniga (2014). De forma similar concluyen Valli Jayanthi et al. (2014) quienes examinaron el género, la edad, la nacionalidad del estudiante, el empleo a tiempo parcial, las actividades extracurriculares y el interés en estudios de posgrado. Esta última referencia introduce la edad como determinante del rendimiento académico. Tejedor (2003) señala que la edad es un factor explicativo del rendimiento, mencionando que dentro de un mismo curso aquellos alumnos que son más jóvenes obtienen un mejor promedio. Coincidiendo con estudios previos de Richardson (1994) y posteriores como el de Valli Jayanthi et al. (2014). En cuanto a la preferencia de elección, los estudios de Yousef $(1991,1993$, y 2011) encontraron que el campo de estudio (contabilidad, administración de empresas, economía, finanzas y ciencias actuariales) influían en el rendimiento académico, así como el campo de estudio en el pre-grado (ciencias o arte). Nosotros examinaremos si la disposición preferencial en la elección de la carrera puede influir en el rendimiento. Respecto a la educación de los progenitores, Ruiz De Miguel (2001) señala que existen factores familiares que ejercen influencia en el rendimiento del alumno, dentro de los más importante se encuentra la educación de los padres, donde aquellos que poseen alta formación académica entregan un mejor clima educativo y una estimulación constante en el estudio de sus hijos, lo que repercute de manera positiva en el rendimiento de los alumnos. Por otro lado, Jara et al. (2008) exponen en su estudio que el colegio donde el alumno terminó la enseñanza media, privado o estatal, es un factor que influye en el rendimiento de éste, mencionando que aquellos que salieron de un colegio privado, de buena calidad y con prestigio dedican más horas de estudio y se adaptan más rápido al ambiente universitario, lo que se traduce en un mejor rendimiento académico, en cambio los alumnos que provienen de colegios estatales dedican menos horas de estudio debido a que no poseen estos hábitos, además tienen una baja autoestima y poseen un bajo nivel socioeconómico, lo que influye de forma negativa en su rendimiento. Ocaña (2011) señala que factores como una mejor base formativa previa a la enseñanza superior, mayor interés (medido en participación y consultas en clases), vocación (medido en preferencia y elección de la carrera) y esfuerzo (medido en asistencia, entrega de trabajos y tareas) de los propios estudiantes influiría positivamente en los resultados académicos del alumno.

Caira y Lescher (2009) realizaron un estudio para determinar el impacto del cambio de plan curricular en estudiantes universitarios, para ello analizaron y compararon cada asignatura con la tasa de aprobación y las calificaciones obtenidas respecto al plan antiguo, obteniendo como resultado una reducción en la tasa de aprobación y un aumento leve en el rendimiento académico de los alumnos. Asimismo, Mlambo (2011) examinó el impacto que tiene el diseño y la planificación del modelo por competencia en el rendimiento, tomando cinco asignaturas comunes, los resultados arrojaron que el modelo incidía positivamente en el rendimiento académico, debido a la mayor comprensión y aprendizaje de los contenidos por parte de los alumnos en las clases teóricas. Otros estudios también han examinado diversos factores de influencia en el rendimiento académico. Por ejemplo, Cabrera y Galán (2003) exploraron la incidencia de la satisfacción escolar del estudiante, o Fernández, Fernández, Álvarez y Martínez (2007), que examinaron la satisfacción con la docencia recibida. Estos ejemplos determinan y justifican el examen sobre el rendimiento académico de un factor como el modelo curricular.

De las evidencias anteriores, se puede entender que el rendimiento académico es un resultado de la influencia de factores del ambiente externo e interno del estudiante, los cuales pueden afectar de manera positiva o negativa en el resultado final. En esta línea, la presente investigación tiene como objetivo estudiar cómo ha influido en el rendimiento académico de los estudiantes la implementación del modelo de formación por competencias, tomando como base el modelo anterior por contenidos en el caso de la carrera Ingeniería Comercial (IC) de la Universidad Católica de la Santísima Concepción (UCSC), basándose en la teoría de los determinantes del rendimiento académico de Tejedor (2003). En este sentido, el estudio buscará dar respuesta a la siguiente hipótesis de investigación, los estudiantes con una formación basada en competencias obtienen mejores resultados académicos en relación al diseño curricular por contenidos. 


\section{METODOLOGÍA}

Se empleó una base de datos donde se encuentra la información del estudiante, referente tanto al ámbito personal como educacional. En total son 852 estudiantes, pertenecientes a primer año la carrera de IC de la UCSC, matriculados durante los años 2007 - 2014. Y se dividen en estudiantes según el modelo de formación por competencia y modelo por contenido. En el primer grupo se encuentran las generaciones del 2007-2010, este grupo está compuesto por 393 alumnos, donde 240 son mujeres y 153 son hombres. En el segundo, están los alumnos que estudiaron bajo el método de formación por competencia, los que pertenecen a las generaciones del 2011-2014, este grupo está compuesto por 459 alumnos, donde 242 son mujeres y 217 son hombres.

Como el objeto del estudio tiene una relación causal, se ha utilizado el método de mínimos cuadrados ordinarios (MCO) a través del cual se estimará una función de regresión lineal que explique si el diseño curricular influye en el rendimiento académico de los alumnos. En el modelo estimado, la variable dependiente es el rendimiento académico de los alumnos al primer año de carrera e identificándose como PPA (promedio ponderado acumulado). Y se han considerado 8 variables explicativas del rendimiento académico del alumno, estas se describen a continuación, incluyéndose el nombre que se le ha dado a cada una para efectos de la estimación del modelo.

1) El diseño curricular del estudiante, que toma valor 1 si el estudiante tuvo una formación por competencia y 0 si fue por contenidos: Plan

2) Género del estudiante, que toma valor 1 femenino y 0 masculino: Gen

3) Edad con la cual el estudiante ingresó a la carrera: Edad

4) Preferencia que le otorgó el estudiante a la carrera al momento de postulación: Pref.

5) Años de educación de la madre: EducM

6) Años de educación del padre: EducP

7) Y 8) Tipo de establecimiento educacional donde el estudiante terminó la educación media: Representado por una variable dummy de 3 atributos: colegios municipal, subvencionado y particular, incorporándose en el modelo solo dos variables para evitar multicolinealidad, C_Sub que toma valor 1 si proviene de un colegio subvencionado y 0 en otro caso, C_Mun que toma valor 1 si proviene de un colegio municipal y 0 en otro caso.

De acuerdo a lo anterior el modelo poblacional a estimar es el siguiente:

$$
\text { PPA }=\beta_{1}+\beta_{2} \text { Plan }+\beta_{3} \text { Gen }+\beta_{4} \text { Edad }+\beta_{5} \text { Pref }+\beta_{6} \text { EducP }+\beta_{7} \text { EducM }+\beta_{8} C \_S u b+\beta_{9} C \_ \text {Mun }+\mu
$$

\section{RESULTADOS}

Como una primera aproximación de los resultados de las variables que influyen en el rendimiento académico de los alumnos se estimaron los coeficientes de correlación entre el PPA y las variables independientes del modelo. La figura 1 muestra en el eje horizontal las variables explicativas ordenadas de mayor a menor según la correlación que tienen con la variable PPA. Como se puede divisar el diseño curricular del estudiante se relaciona de manera directa y es la que mayor grado de correlación tiene, sin embargo como esta es una variable dummy en la estimación del modelo que viene a continuación se determinara si es el modelo por contenidos o la formación por competencia el que influye con mayor grado en el rendimiento académico del alumno.

Se puede observar también que el género se relaciona de manera directa y es el segundo con mayor correlación. En cuando a las variables que representan el colegio del cual proviene el estudiante, hay una disyuntiva, puesto que el colegio municipal se relaciona de forma positiva, en cambio el colegio particular y subvencionado tienen relación inversa. También se observa que la educación de los padres tiene una relación inversa. Al igual que la edad, esto implica que aquellos alumnos que entran con menor edad a la carrera obtienen mejores rendimientos académicos, al igual que aquellos que escogen como su primera opción la carrera al momento de postular.

La tabla 2 muestra la estimación de la función de regresión lineal, a través de $M C O$, indicada en la metodología. En compañía del coeficiente aparece marcada con asterisco la significancia de la variable: *No significativa, ${ }^{* *}$ Significativa, ${ }^{* * *}$ Significativa menor al $1 \%$. De acuerdo a ello, se encontró que existe una diferencia significativa en el rendimiento académico entre aquellos alumnos que estudiaron bajo el modelo tradicional y los que estudiaron bajo el modelo por competencia. Estos resultados concuerdan con los 
obtenidos por otros estudios que señalan que el diseño curricular influye en el rendimiento académico de los alumnos como los de Caira y Lescher (2009).

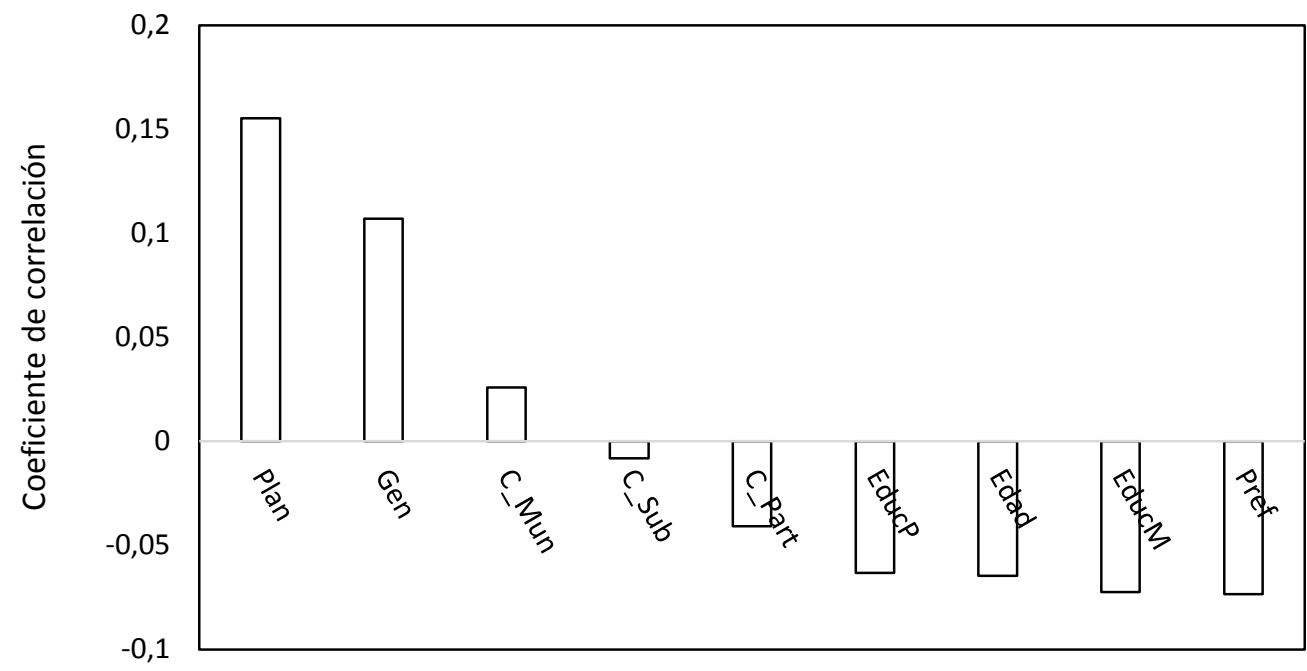

Fig. 1: Correlación entre el promedio ponderado y las variables independientes

En cuanto a las demás variables incorporadas en el estudio, a continuación se mencionan en orden de importancia las que son significativas y por ende influyen en el rendimiento académico de los alumnos; género del estudiante, donde se refleja que las mujeres obtienen mejores resultados académicos que los hombres, lo que concuerda con los resultados obtenidos por Soria y Zúñiga (2014); la edad con la cual entra el estudiante a la carrera, se indica que a menor edad mejor resultado académico, esto apoya la investigación de Tejedor (2003) quien señala que en un mismo curso aquellos estudiantes de menor edad obtienen un mejor rendimiento; la preferencia que le otorga el estudiante a la carrera cuando postula a ella, donde se señala que entre más cercana a uno sea la preferencia mejor es el promedio, esto apoya la línea investigativa de Ocaña (2011), quienes señalan que la preferencia que le otorga el alumno a la carrera influye en el alto rendimiento de éste. Por otra parte, se detectó que la educación de los padres y el colegio del cual proviene el alumno no influyen en su rendimiento académico.

Tabla 2: Estimación del modelo a través de MCO, donde la variable dependiente es PPA

\begin{tabular}{|l|l|c|c|}
\hline Variable & \multicolumn{1}{|c|}{ Coef. } & Desv. Típica & Media \\
\hline Const & 5,02779 & 0,326055 & \\
\hline Plan & $0,24711^{* * *}$ & 0,050964 & 0,5387323 \\
\hline Pref & $-0,03371^{* *}$ & 0,019051 & 2,2147887 \\
\hline Gen & $0,16643^{* * *}$ & 0,051049 & 0,5657277 \\
\hline Edad & $-0,02888^{* *}$ & 0,015158 & 18,604460 \\
\hline C_Sub & $0,08214^{*}$ & 0,119981 & 0,4260563 \\
\hline C_Mun & $0,10396^{*}$ & 0,121313 & 0,5234741 \\
\hline EducP & $-0,00322^{*}$ & 0,005185 & 9,4870892 \\
\hline EducM & $-0,00909^{*}$ & 0,005974 & 9,9143192 \\
\hline
\end{tabular}

En la tabla 3 se presenta la estimación del test de chow, el cual se le realizó a la variable Plan para verificar los datos obtenidos anteriormente. Como se aprecia en el estadístico de contraste de chow, existe diferencia estructural en el rendimiento académico de los alumnos que estudian bajo el modelo por competencia en comparación a los que estudiaron bajo el método tradicional por contenido.

Los datos se obtuvieron del alumnado de la carrera de IC de la UCSC, en este contexto el impacto del modelo por competencia solo se pudo analizar con el rendimiento académico, ya que no se contaba con otra información de los alumnos que pudiera representar como ha impactado en ellos el nuevo modelo, esto se debe a que el modelo es reciente en la carrera, lo que limita la información. Es por ello que los resultados no pueden extrapolarse a otros ámbitos de estudio u otras culturas, ya que pueden existir diferencias entre un contexto y otros, tanto en los alumnos como en la universidad en sí. 
Tabla 3: Test de chow

\begin{tabular}{|l|r|c|c|}
\hline \multicolumn{1}{|c|}{ variable } & Coef. & Desv Tipica & Valor $p$ \\
\hline const & 4,01168 & 0,48071 & 0,00000 \\
\hline Pref & $-0,07659$ & 0,02712 & 0,00480 \\
\hline Gen & 0,20109 & 0,07568 & 0,00800 \\
\hline Edad & 0,04103 & 0,02211 & 0,06390 \\
\hline C_Sub & $-0,137291$ & 0,18592 & 0,46040 \\
\hline C_Mun & $-0,07164$ & 0,18503 & 0,69870 \\
\hline EducP & $-0,00816$ & 0,00768 & 0,28800 \\
\hline EducM & $-0,00610$ & 0,00850 & 0,47320 \\
\hline Plan & 2,15020 & 0,64790 & 0,00090 \\
\hline PI_Pref & 0,07275 & 0,03778 & 0,05450 \\
\hline PI_Gen & $-0,07909$ & 0,10181 & 0,43740 \\
\hline PI_Ed & $-0,128579$ & 0,03015 & 0,00002 \\
\hline PI_C_Sub & 0,37492 & 0,24208 & 0,12180 \\
\hline PI_C_Mun & 0,30030 & 0,24391 & 0,21860 \\
\hline PI_EducP & 0,00963 & 0,01035 & 0,35230 \\
\hline PI_EducM & $-0,00390$ & 0,01187 & 0,74210 \\
\hline Contraste de Chow de diferencia estructural con respecto a Plan \\
F(8,836)=6,40006 con valor $\mathrm{p} 0,0000$ & \\
\hline
\end{tabular}

\section{CONCLUSIONES}

De acuerdo a la investigación realizada el objetivo se ha cumplido y la hipótesis formulada ha sido verificada, ya que la estimación de la función de regresión lineal a través de MCO arrojó que sí existe una diferencia en el rendimiento académico de los alumnos que estudian bajo el modelo por competencia en comparación con aquellos que estudian con el modelo por contenido. En confirmación a ello a continuación se presentan las siguientes conclusiones finales del estudio.

Existe una diferencia significativa en el rendimiento académico de los alumnos dependiendo del diseño curricular utilizado, donde aquellas personas que tienen una formación educacional por competencia obtienen un mejor rendimiento académico en comparación a quienes tienen una formación tradicional. Estos datos indican que el nuevo modelo formativo por competencia, cuya metodología está enfocada en el estudiante buscando que éste relacione teoría con práctica, ha tenido un efecto positivo para los alumnos, impactando en sus calificaciones.

En cuanto a los demás datos encontrados, se detectó que las mujeres obtienen un mejor rendimiento académico en la carrera IC que los hombres. Por otro lado, existe una correlación positiva entre la edad del estudiante al ingresar a la carrera y su rendimiento académico. Así, la probabilidad de obtener un mejor rendimiento académico aumenta al incrementarse la edad de ingreso del estudiante. Finalmente aquellos alumnos que al momento de postular a las universidades escogieron a la carrera de IC entre sus primeras opciones tienen mayores probalidades de obtener un mejor rendimiento académico.

Este estudio sirve como respuesta a la duda existente entre el profesorado y alumnado de la carrera de IC de la UCSC, de que si efectivamente todo el cambio que se ha realizado en temas de currículo, enseñanza, metodología de evaluaciones, ha tenido un impacto positivo en los alumnos, es decir, si estos logran una mejor relación entre la teoría y la práctica. Los resultados demuestran que el impacto ha sido positivo, esto indica que la implementación del nuevo modelo ha cumplido con su objetivo.

\section{REFERENCIAS}

Arnaz, J., La Planeación Curricular, 2ª edición. Trillas, México (1993)

Cabrera, P., y Galán, E., Satisfacción Escolar y Rendimiento Académico, Revista de Psicodidáctica: 14, 87-9 (2003) 
Caira, N., y Lescher, I. Impacto de la reforma del plan curricular en el rendimiento académico, Revista de Ciencias Sociales: 16(2), 280 - 291 (2009)

$\mathrm{CRUCH}$, Consejo de Rectores de las Universidades Chilenas, Innovación curricular en las universidades del consejo de rectores, $1^{\circ}$ edición, 1-190, Nuevamérica impresiones, Chile (2012)

Borde, S. F., Predictors of Student Academic Performance in the Introductory Marketing Course, Journal of Education for Business: 73, 302-306, (1998)

Bustamante, M. A., Oyarzún, C. H., Grandón, M. L., y Abarza, C. G., Fundamentos de la Enseñanza por Competencias a Nivel de Postgrado en dos Universidades Públicas Chilenas, Formación Universitaria: 8(6), 23-30 (2015)

Fernández Rico, J. E., Fernández Fernández, S., Álvarez Suárez, A., y Martínez Camblor, P., Éxito Académico y Satisfacción de Estudiantes con la Enseñanza Universitaria, Relieve: 13(2), (2007)

Guzmán Droguett, M. A., Maureira Cabrera, Ó., Sánchez Guzmán, A., y Vergara González, A., Innovación Curricular en la Educación Superior: ¿Cómo se Gestionan las Políticas de Innovación en los (Re)Diseños de las Carreras de Pregrado en Chile?, Perfiles Educativos: 37(149), 60-73 (2015)

Jara, D., Velarde, H., Gordillo, G., Guerra, G., León, I., Arroyo, C. y Figueroa, M., Factores influyentes en el rendimiento académico de estudiantes del primer año de medicina, Anales de la Facultad de Medicina: 69(3), 103-197 (2008)

Martínez-Clares, P., Martínez-Juárez, M y Muñoz-Cantero, J.M., Formación Basada en Competencias en Educación Sanitaria: Aproximaciones a Enfoques y Modelos de Competencia, Relieve: 14(2), 1-23 (2008)

Mlambo, V., An Analysis of Some Factors Affecting Student Academic Performance in an Introductory Biochemistry Course at the University of the West Indies, Caribbean Teaching Scholar: 1(2), 79-92 (2011)

Navarro, R., El Rendimiento Académico: Concepto, Investigación y Desarrollo, Reice: 1(2), 1-15 (2003)

Ocaña, Y., Variables Académicas que Influyen en el Rendimiento Académico de los Estudiantes Universitarios, Revista de investigación educativa: 15(27), 165-179 (2011)

Organización de las Naciones Unidas para la Educación, la Ciencia y la Cultura, Conferencia Mundial sobre la Educación Superior, (Tomo I) (1998). http://unesdoc.unesco.org/images/0011/001163/116345s.pdf

Palominos, F.E., Mendez, M.A. y Barrera, R.A., Sistema de Perfeccionamiento Orientado a Competencias para Docentes de la Educación Superior, Formación Universitaria, 7(3), 11-22 (2014)

Richardson, J.T.E., Mature Students in Higher Education: Academic Performance and Intellectual Ability, Higher Education: 28(3), 373-386 (1994)

Riesco, M., El Enfoque por Competencias en el EEES y sus Implicaciones en la Enseñanza y el Aprendizaje, Tendencias Pedagógicas: 13, 79-105 (2008)

Ruiz de Miguel, C., Factores familiares vinculados al bajo rendimiento, Revista Complutense de Educación: 12(1), 81-113 (2001)

Schwab, J. The practical 4: Something for Curriculum Professors to Do, Curriculum Inquiry: 13, 239-266 (1983)

Soria, K. y Zúñiga, S., Aspectos Determinantes del Éxito Académico de Estudiantes Universitarios, Formación Universitaria: 7(5), 41-50 (2014)

Taba, H. La Elaboración del Currículo. Troquel, Argentina (1974)

Tardif, J., Développer un programme par compétences: de l'intention à la mise en oeuvre, Pedagogie Collegiale: 16(3), 36-44 (2003)

Tejada, J., El docente universitario ante los nuevos escenarios: implicaciones para la innovación docente, Acción Pedagógica: 11(2), 30-42. (2002)

Tejedor, J., Poder explicativo de algunos determinantes del rendimiento en los estudios universitarios, Revista española de pedagogía: 224, 5-32 (2003) 
Tyler, F.T., Teacher Personalities and Teaching Competencies, The Social Review: 64(8), 429-449 (1960)

UNESCO, Declaración Mundial sobre la educación Superior en el siglo XXI: Visión y Acción (en la web: htpp:/ /www.unesco.org, acceso 21 de diciembre 2015), UNESCO (1998)

Valli Jayanthi, S., Balakrishnan, S., Lim Siok Ching, A., Aaqilah Abdul Latiff, N., \& Nasirudeen, A. M. A. Factors Contributing to Academic Performance of Students in a Tertiary Institution in Singapore. http://doi.org/10.12691/education-2-9-8, American Journal of Educational Research: 2(9), 752-758 (2014)

Vélez, G. y Terán, L., Modelos para el Diseño Curricular, Pampedia: (6), 55-65 (2009)

Vidal, M. y Pernas, M., Diseño Curricular, Educación Médica Superior: 21(2), 1-10 (2007)

Villa, A. y Poblete, M., Aprendizaje basado en competencias, $1^{\circ}$ edición, 1-333. Ediciones Mensajero, Bilbao (2007)

Walker, D. F., Fundamentals of Curriculum. Harcourt Brace jovanovich, San Diego (1990)

Woodfield, R., y Earl-Novell, S., An Assessment of the Extent to Which Subject Variation in Relation to the Award of First Class Degree Between the Arts and Sciences Can Explain the 'Gender Gap', British Journal of Sociology of Education: 27(3), 355-372 (2006)

Yousef, D. A., Study of the Effect Of Academic and Demographic Factors on Students' Performance in Operations Research Course with Special Reference to the Students of the Faculty of Economics \& Administrative Science (The UAE University), Journal of Social Affairs: 8, 67-88 (1991)

Yousef, D. A., Students' Attitudes Towards Quantitative Courses: The Faculty of Economics \& Administrative Science: The UAE University, Journal of Administrative Sciences and Economics: 4, 287-318 (1993)

Yousef, D. A., Academic Performance of Business Students in Quantitative Courses: A Study in the Faculty of Business and Economics at the UAE University, Journal of Innovative Education: 9(2), 255-267 (2011) 